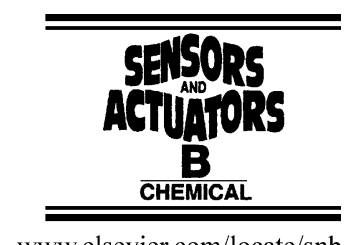

www.elsevier.com/locate/snb

\title{
A silicon microfluidic ultrasonic separator
}

\author{
N.R. Harris ${ }^{\mathrm{a}, *}$, M. Hill $^{\mathrm{b}}$, S. Beeby ${ }^{\mathrm{a}}$, Y. Shen ${ }^{\mathrm{b}}{ }^{\text {, N.M. White }}{ }^{\mathrm{a}}$, \\ J.J. Hawkes ${ }^{\mathrm{c}}$, W.T. Coakley ${ }^{\mathrm{c}}$ \\ a Department of Electronics and Computer Science, University of Southampton, Southampton SO17 1BJ, UK \\ ${ }^{\mathrm{b}}$ School of Engineering Sciences, University of Southampton, Southampton SO17 1BJ, UK

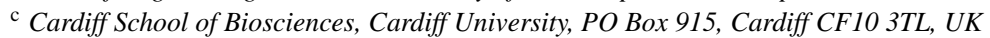

\begin{abstract}
Ultrasonic standing waves can be used to generate forces on particles within a fluid. Such forces have a number of potential applications in microfluidic devices. This paper describes a device that provides filtration on a microfluidic scale. It is microfabricated and uses ultrasound in the megahertz frequency range to concentrate particles at a node within the flow. It offers the possibility of a functional equivalent of a centrifugal separator for microfluidic systems. It is constructed using silicon and Pyrex, and hence is compatible with established microfabrication techniques. The modelling, design, fabrication and control of the device are discussed.
\end{abstract}

(c) 2003 Elsevier B.V. All rights reserved.

Keywords: Microfluidic; Microengineering; Ultrasonic

\section{Introduction}

High frequency, acoustic standing waves can be used to separate materials with different acoustic impedances. The technique can be used to agglomerate particles, to hold particles within a flow, or to manipulate particles with the flow [1]. The device described in this paper falls into the latter category, as it is a microfluidic flow through separator, and is an extension of previous work performed by Hawkes and Coakley [2] into similar devices fabricated using traditional rather than microfabrication techniques. The device is fabricated in silicon and Pyrex and, as such, is able to exploit standard silicon processes. Driving is achieved via a bonded PZT plate, although future developments will use a thick-film PZT actuator [3]. Fig. 1 shows a schematic cross-section of the prototype.

\section{Principle of operation}

A fluid/particle mixture is drawn into the device through the port on the left of Fig. 1. An acoustic standing wave, of a half wavelength, is maintained within the cavity and as the particles move through the field they migrate to the pressure node at the centre of the cavity as is shown by the idealised pressure profile in Fig. 2. A fraction of the particle-free fluid

\footnotetext{
* Corresponding author. Tel.: +44-2380-593-667.

E-mail address: nrh@ecs.soton.ac.uk (N.R. Harris).
}

can then be drawn from the "clean" outlet and the remainder of the fluid/particle mixture can be drawn from the final, dirty outlet. Such a scheme allows for a relatively small quantity of clarified fluid to be produced. Different geometries have the potential to increase the proportion of clarified fluid recovered, or alternatively to increase the concentration of the particles recovered from the "dirty" outlet. A separator with the ability to clarify samples or concentrate particles within a sample has important potential applications in integrated microfluidic sensing systems.

\section{Modelling}

Previous work [4] has allowed the characterisation and validation of a multi-layered resonant structure, constructed in stainless steel, and this has been extended to include the silicon/Pyrex construction described here. Amongst important variables that can be adjusted are:

- the depth of the cavity,

- the thickness of the Pyrex layer,

- the thickness of the silicon layer.

The basic structure modelled is shown in Fig. 3 and consists of several layers, whose physical parameters can be altered according to the structure being modelled, and is composed of a piezoelectric transducer, a layer of adhesive, a silicon layer, a filtration chamber filled with fluid (in this case water), and a Pyrex reflector plate. The parameters of 


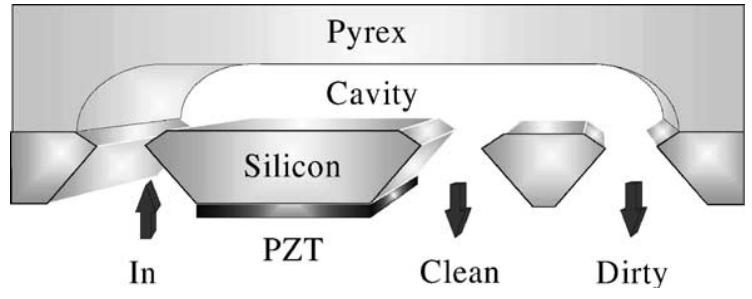

Fig. 1. Cross-section of the device.

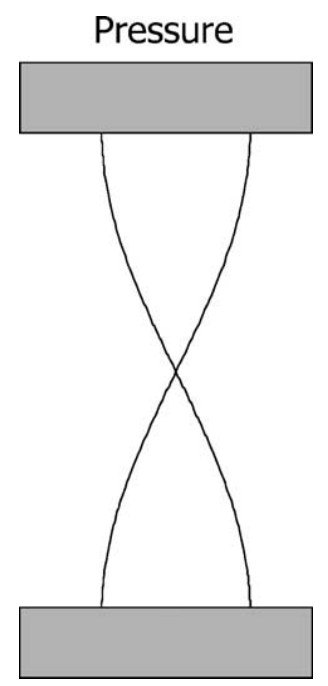

Fig. 2. Pressure profile through half wavelength cavity with rigid boundaries.

the layers can be altered, according to the structure being modelled. For example, changing the parameters of the fluid layer to those of air result in a model of an empty cell, which is useful for isolating the effects of the PZT and silicon layers. The model assumes that the concentration of particles is not high enough to disrupt the acoustic field significantly.

The currently available outputs from the model are:

- impedance seen looking into the device,

- voltage seen when driven from a $50 \Omega$ driving stage,

- acoustic force,
- stored energy,

- performance number [1].

\section{Verification of the model}

Verification of the model is an important part of the work. Data produced by Hawkes and Coakley [2] have shown experimentally the separator performance (clearance) of the stainless steel chamber against frequency. Modelling, using the method described by Hill and Wood [5] has demonstrated that a good indication of actual filtration performance can be predicted by the product of the acoustic energy stored in the fluid and the driving frequency (the "energy frequency product") [4]. The model was verified against the stainless steel cell, with data supplied from a working separator tested at Cardiff University. Fig. 4 shows the correlation of the experimental and predicted results for the stainless steel cell.

This gives confidence that the model approach is valid, if only for stainless steel construction. It is, however, still important to verify the model with silicon and Pyrex and because many of the acoustic figure of merit parameters (such as stored energy and performance number) are not directly accessible, validation has to be undertaken by comparing the accessible electrical parameters (such as measured voltage or impedance) with an experimental device. Before any validation can be achieved, a reference device has to be constructed, and so a best estimate of key parameters was made and model predictions were undertaken.

Modelling of the system, as described by Hill and Wood [5], has indicated that the most critical parameter in determining the operating frequency of the device is the depth of the cavity. In order to allow direct comparison with the performance of the stainless steel filter described by Hawkes and Coakley [2], the target cavity depth was $250 \mu \mathrm{m}$, to allow a nominal half-wave resonance of $3 \mathrm{MHz}$. Modelling was carried out to establish the optimum thicknesses for the remaining parameters. In order to reduce the complexity, and therefore increase the ease of manufacture, modelling work concentrated on using standard thicknesses of materials where possible. This work indicated that the cell would work well using a standard thickness silicon matching layer

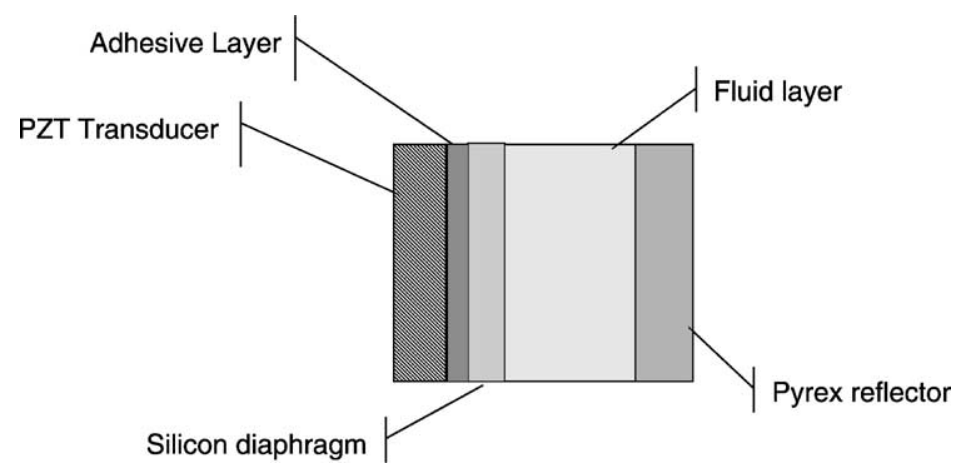

Fig. 3. Basic structure for modelling. 


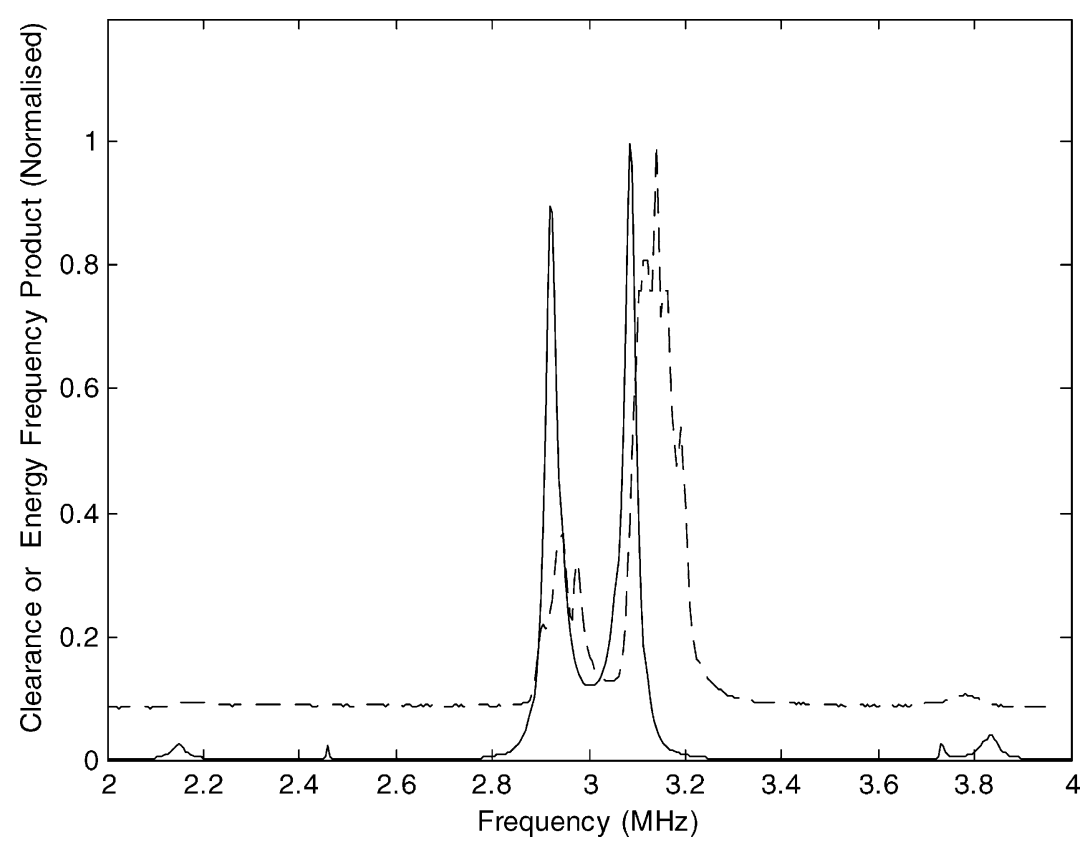

Fig. 4. Comparison of experimental (dotted) and modelled data for the Cardiff stainless steel cell.

of $525 \mu \mathrm{m}$, when used with a standard thickness Pyrex wafer of $1.7 \mathrm{~mm}$ with a cavity depth of $240 \mu \mathrm{m}$. To illustrate the effect that cavity depth has on the predicted performance, Fig. 5 should be considered.

This shows the variation of the stored energy frequency product as a function of cavity depth, when the total thickness of both Pyrex and cavity is fixed at $1.7 \mathrm{~mm}$. As can be seen, the stored energy frequency product (a measure of the energy stored in the standing wave) reaches a peak at about $240 \mu \mathrm{m}$. With a frequency of $3 \mathrm{MHz}$ and cavity lateral di- mensions of $5 \mathrm{~mm}$ wide by $12 \mathrm{~mm}$ long, this equates to an energy density of about $44 \mathrm{~J} / \mathrm{m}^{3}$.

In comparison, modelling changes in the thickness of the silicon matching layer whilst using the previously defined Pyrex construction has demonstrated that the performance of the device will be effectively insensitive to manufacturing tolerances in the silicon wafer. Wafers typically have a tolerance of $\pm 50 \mu \mathrm{m}$ in a nominal thickness of $525 \mu \mathrm{m}$, for example. For such a wafer, the stored energy frequency product varies by about $5 \%$.

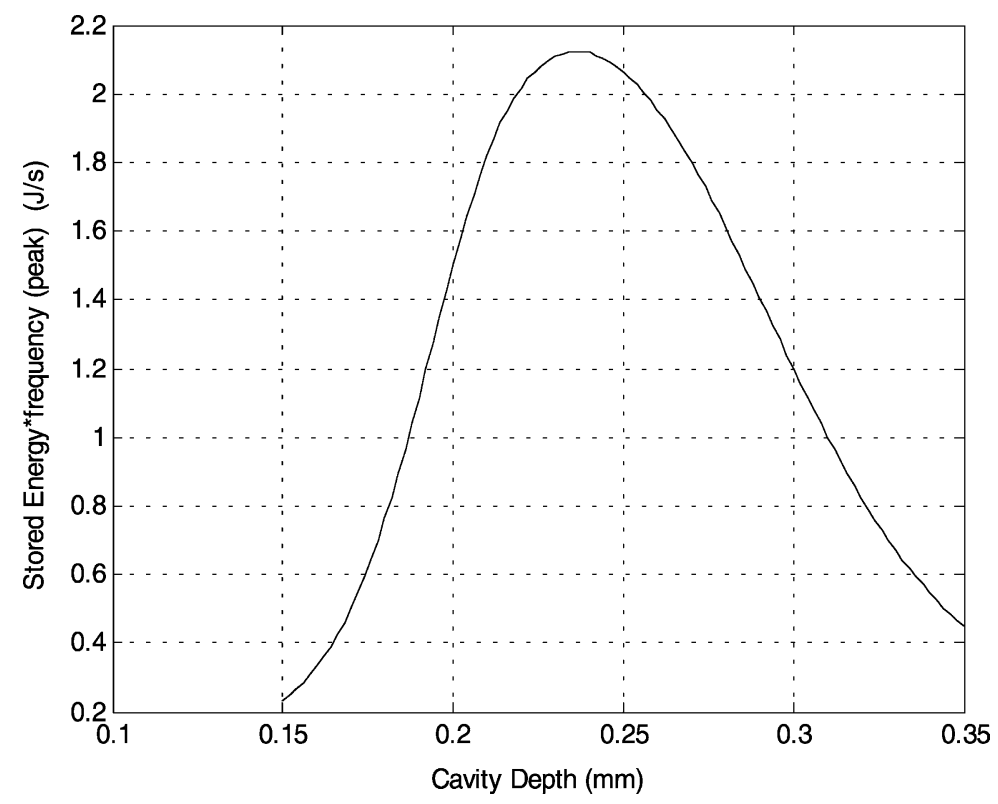

Fig. 5. Variation of performance with cavity depth. 


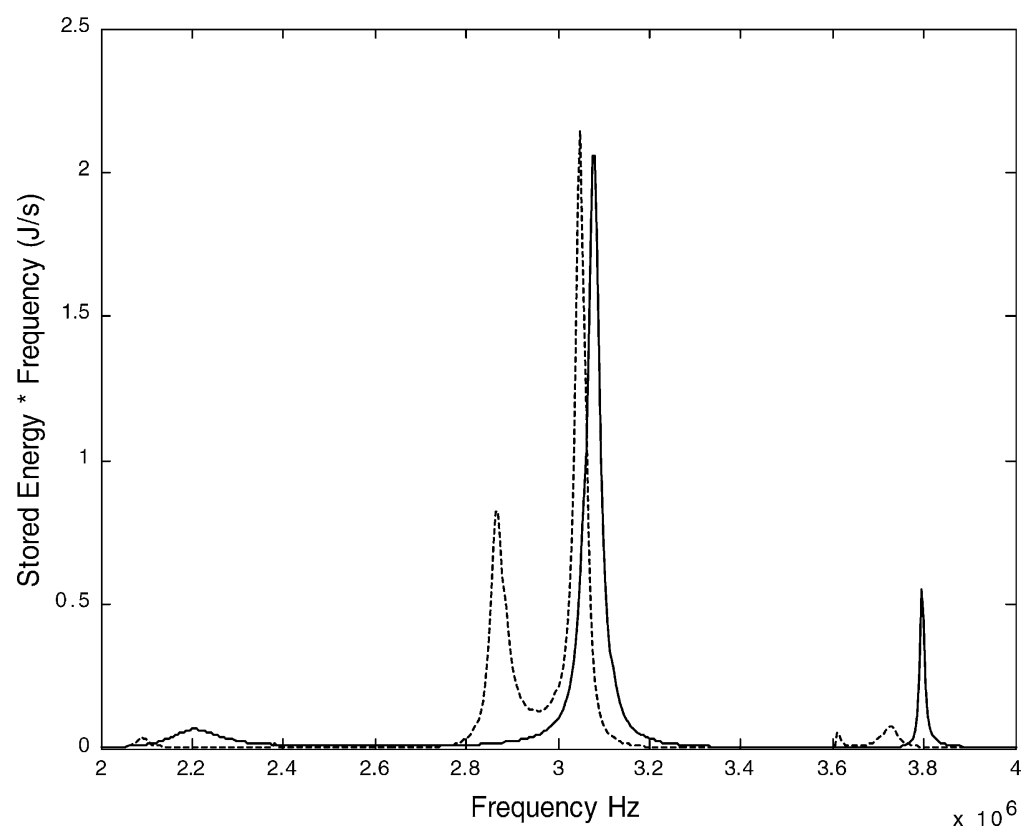

Fig. 6. Comparison of the performance of stainless steel (dashed) and silicon devices.

If the stored energy frequency curves are compared with the original stainless steel cell we can see that the curve for the silicon device has just one dominant peak (Fig. 6). Despite a wide tolerance on peak magnitudes due to uncertainties in material parameters, these simulated results suggest that the silicon-fabricated device should perform at least as well as the steel chamber.

\section{Construction}

Two types of device were fabricated; one with double chamfered ports and one with single chamfers. In order to achieve this, double-sided wafer processing was required. This was achieved using a double-sided alignment process and standard wet $\mathrm{KOH}$ etching leaving chamfers at the angle of $54.7^{\circ}$. The chamber was then formed by etching a $1.7 \mathrm{~mm}$ thick Pyrex wafer to the desired depth of $240 \mu \mathrm{m}$. This was achieved with a $30 \mathrm{~min}$ etch in $48 \%$ buffered hydrofluoric acid using a chrome/gold mask (50 and $500 \mathrm{~nm}$ thick, respectively) and Shipley S1818 resist in order to minimise pinholes. The silicon and Pyrex wafer were then anodically bonded together. Fig. 6 shows a SEM of a single chamfer port, and the section of the Pyrex cavity. These two different port profiles were selected because although the single chamfered port is easier to manufacture, there may be some fluid flow advantages in the double chamfered profile. Future experimental work will address this aspect, although some initial computational fluid dynamic results are reported by Hill et al. [6]. Fig. 7 shows a SEM of a single chamfer port, and a section of the Pyrex cavity, while Fig. 8 shows a photograph of the finished separator mounted on a Perspex manifold, to allow easy connection of pipe work.

\section{Impedance measurements}

In order to verify the validity of the model against the fabricated device, an impedance analyser was used. The instrument used was a Hewlett Packard 4192A frequency analyser, under PC control. Initially, measurements were taken with both 0.5 and $1 \mathrm{~V}$ excitation amplitudes. As no measurable difference was noted, all subsequent measurements were taken at an excitation voltage of $1 \mathrm{~V}$.

The approach taken was to verify the model a layer at a time in order to reduce the number of degrees of freedom, and thus uncertainty in the various parameters of the layers. The first part of the model that required refinement was the response of the PZT driving element in isolation, and its impedance trace was taken. The transducer model parameters were then adjusted to give a good match. The graph (Fig. 9) shows the match obtained. It is very important to get the transducer data as close as possible to the experimental results as this is effectively the input to the model system, and so has a profound effect on the output data.

The next layer in the model is the glue layer. This has an uncertainty associated with it, as the acoustic and material parameters of the glue have to be estimated. It is possible to reduce the uncertainty in the layer thickness by the simple expedient of sectioning another device and measuring the thickness using the SEM. This was duely done and the layer was measured to be between 8 and $10 \mu \mathrm{m}$.

It is therefore possible to refine the effect of the combined silicon and glue layer. For this, an impedance trace of the cell filled with air was taken and compared with the model. The results are shown in Fig. 10. A good match between the measured and modelled impedance is evident. 

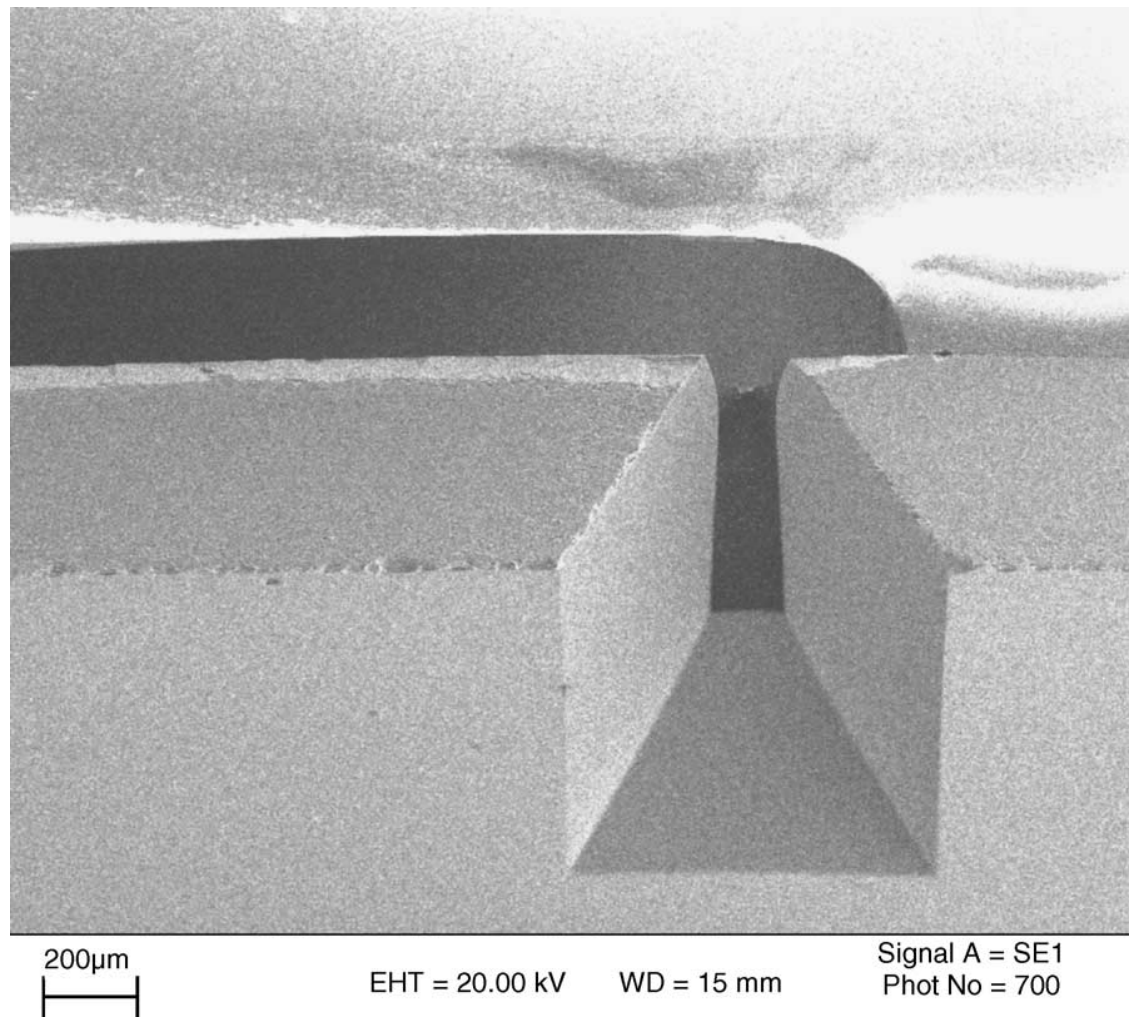

Fig. 7. SEM of a single chamfer port.

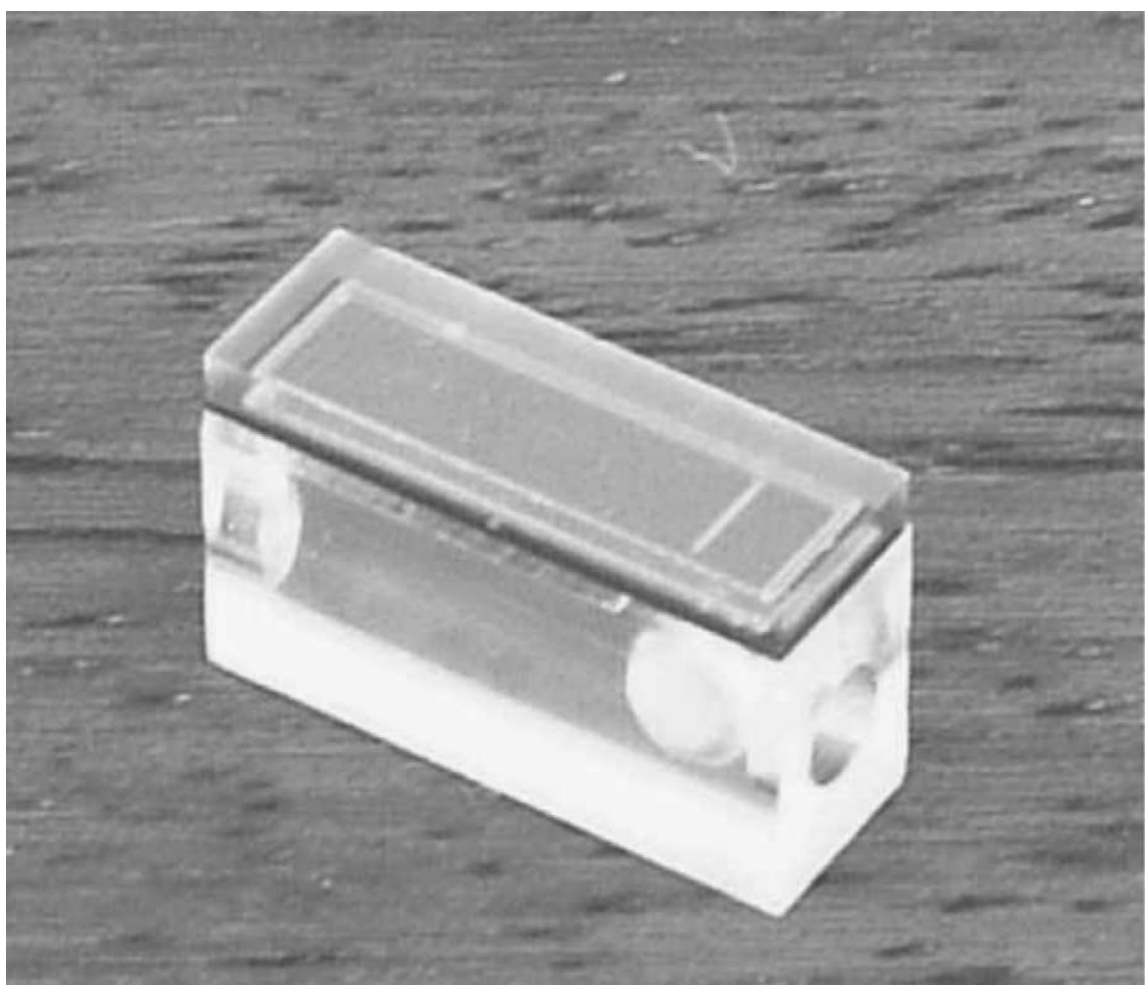

Fig. 8. The assembled device. 


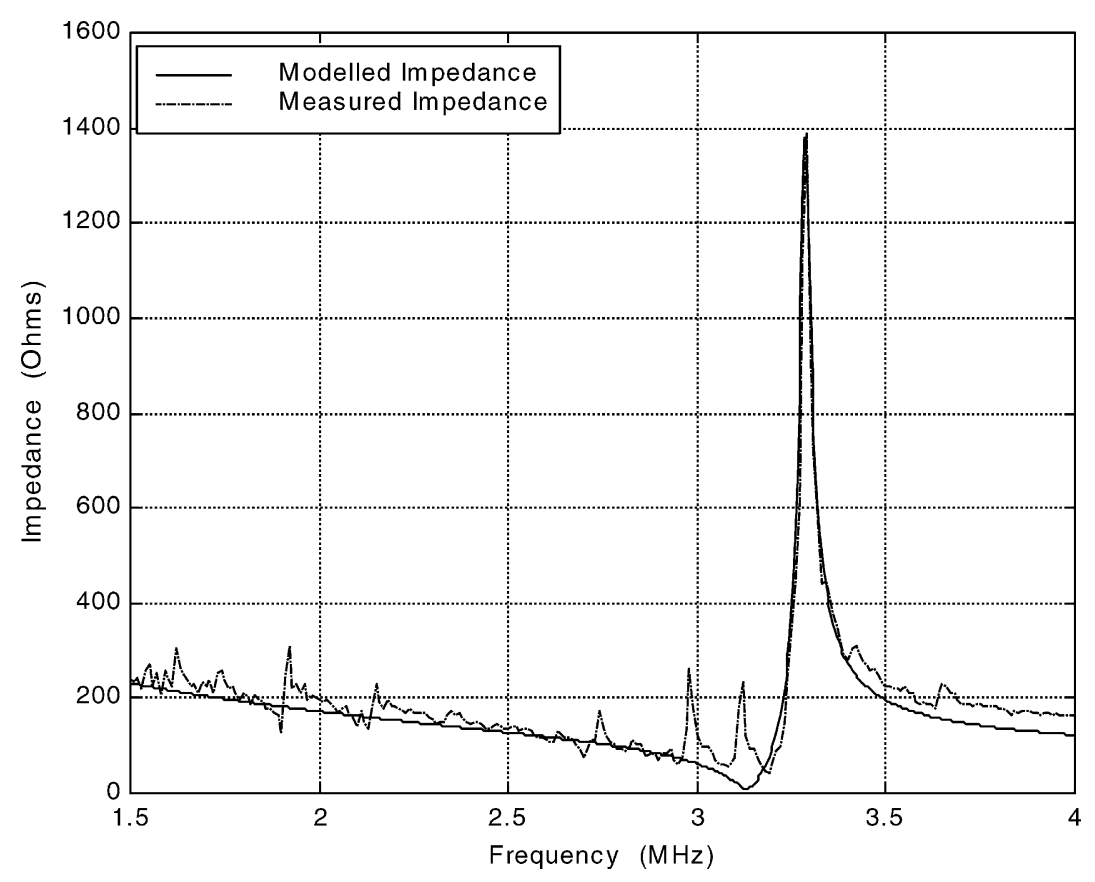

Fig. 9. Modelled (solid) and measured (dashed) results for the transducer in isolation.

The next part of the model is the cavity, which is made significant by introducing a fluid such as water into it, rather than having it filled with air. The key physical parameter that can be measured directly is the cavity depth. This was checked with an optical, non-contact form measurement system, which indicated that the cavity had been over-etched to an average of about $290 \mu \mathrm{m}$ and was not entirely flat, and so the cavity resonance is expected to be lower than the origi- nal predicted value as indicated in Fig. 5. The depth profile is shown in Fig. 11.

The device was filled with water and impedance measurements were taken. Again the modified predicted and measured results are shown (Fig. 12), and a good match is indicated, although it must be noted that the acoustic properties of the fluid may vary and this will have an effect. For example, temperature will have an effect as it alters the

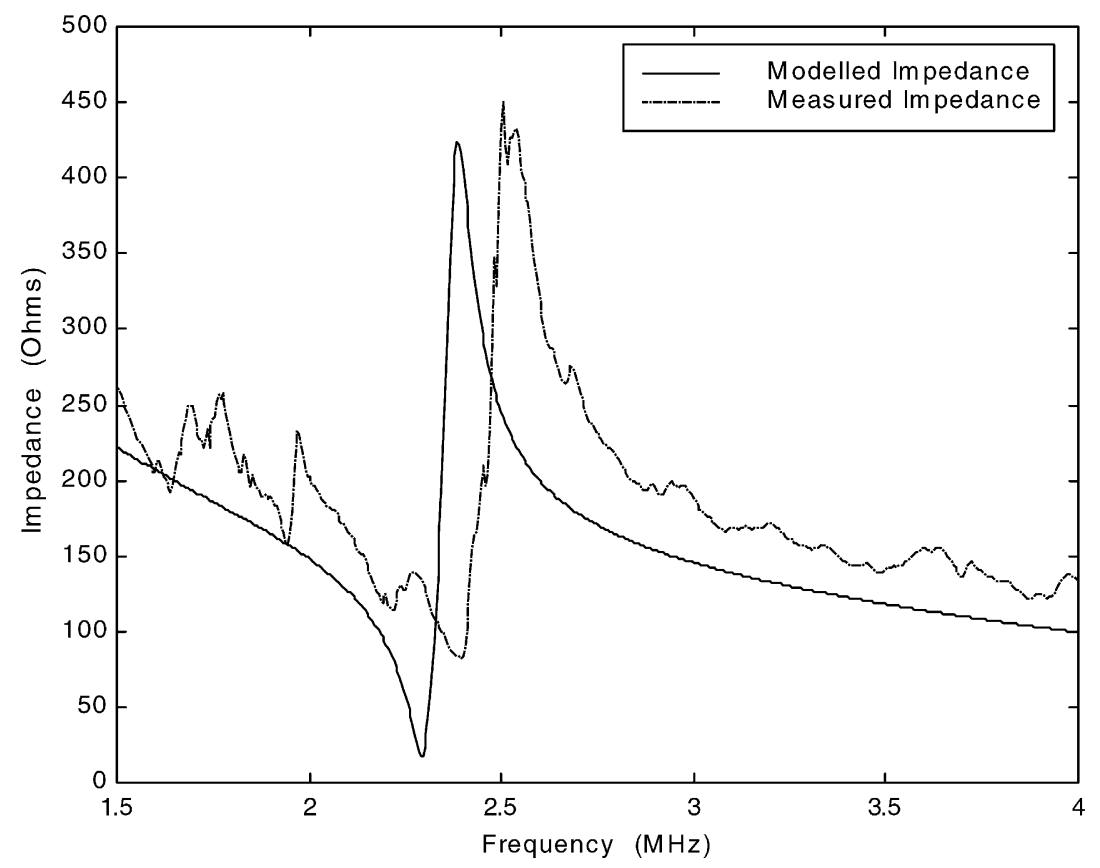

Fig. 10. Modelled (solid) and measured (dashed) results for the transducer and silicon layer. 

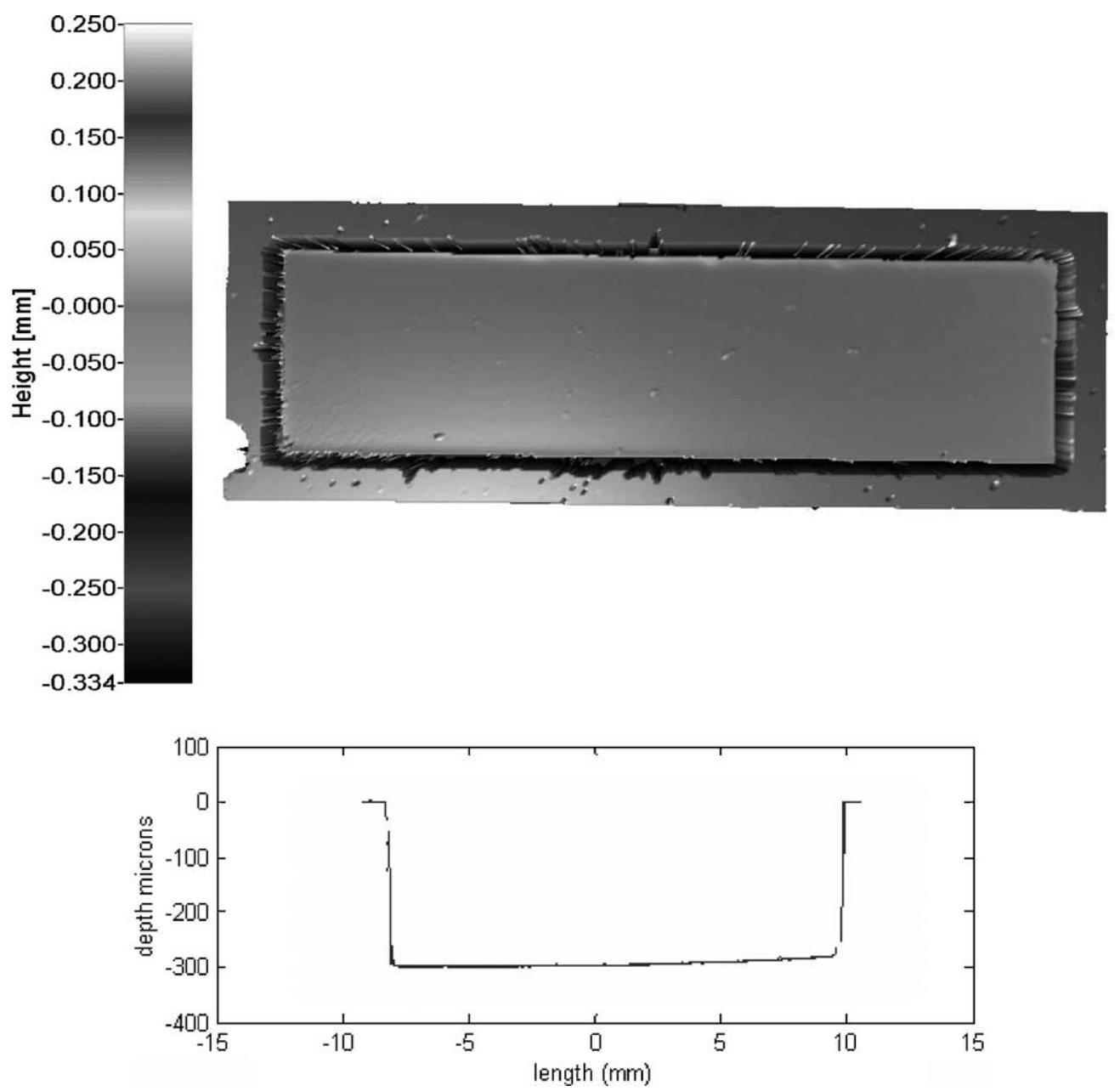

Fig. 11. Depth profile of the cavity.

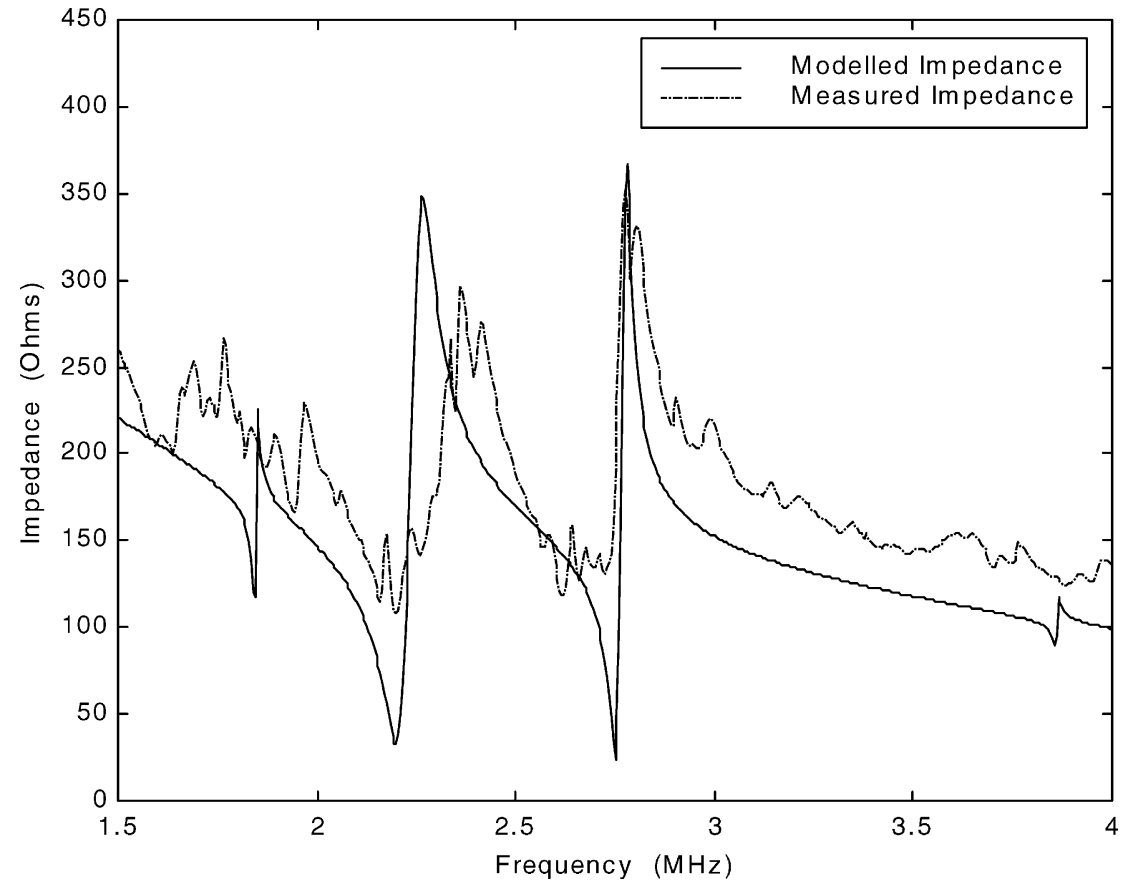

Fig. 12. Complete cell including fluid and Pyrex layers. 


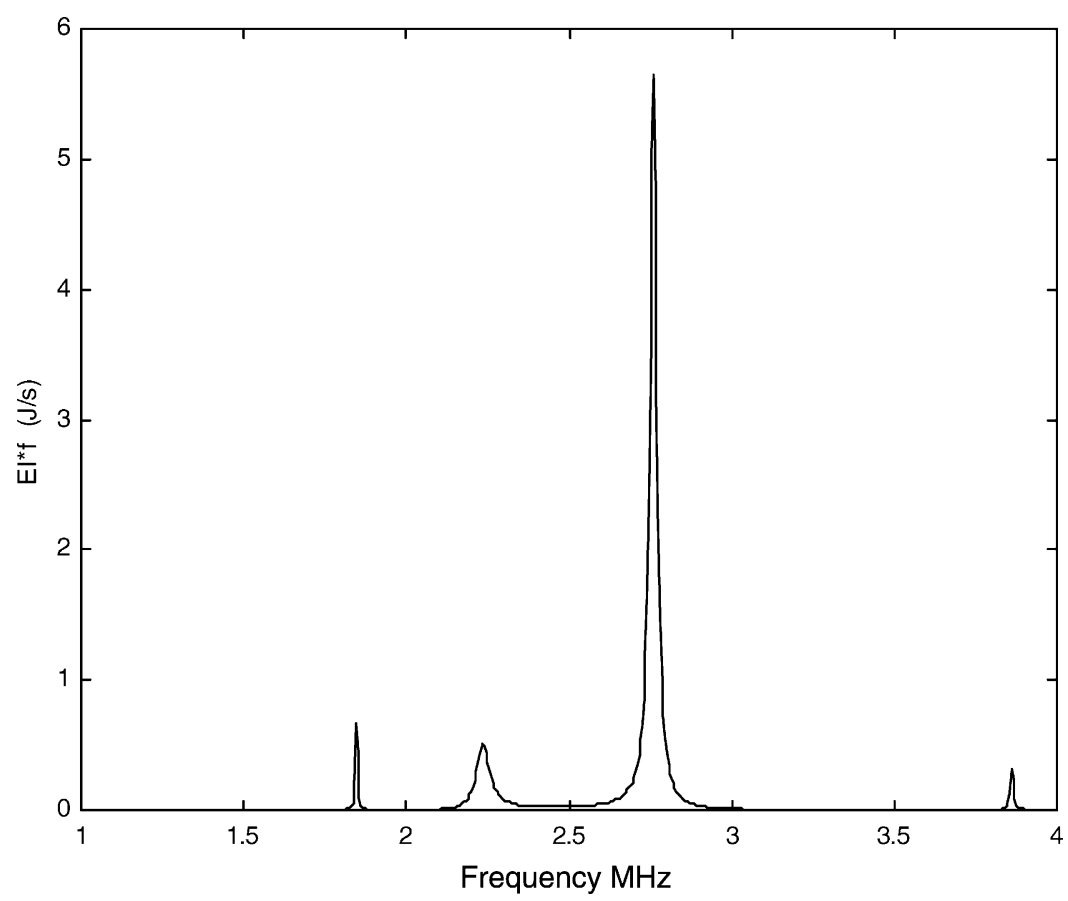

Fig. 13. Predicted separator performance.

\section{$\underline{\text { Digital Controller }}$}

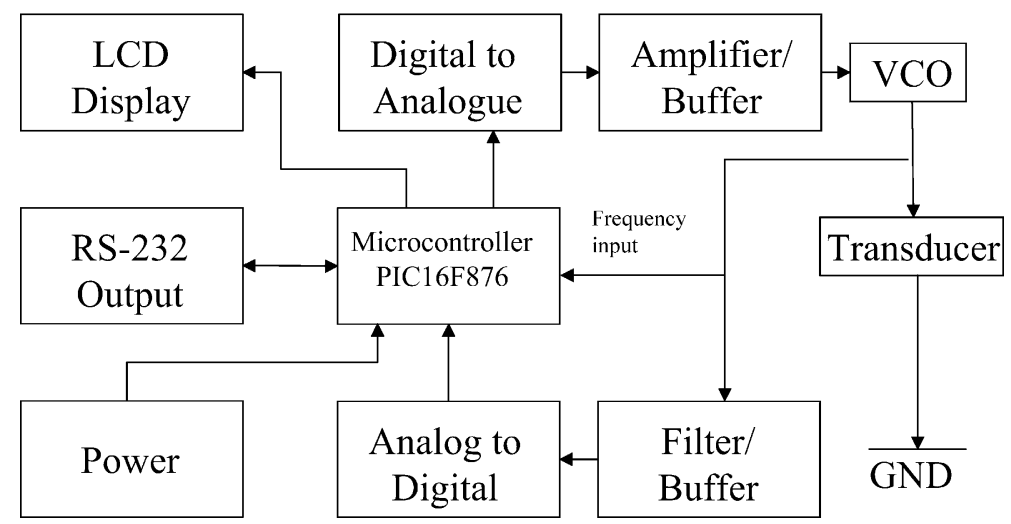

Fig. 14. Controller system diagram.

fluid's acoustic properties, as will the concentration of the particles to be separated. This trace is the response of the complete cell, because as soon as the cavity is brought into play, the Pyrex backing layer also has to be included.

The main feature to note is that the trace now shows two clear peaks. The higher frequency peak is due to the cavity resonance and the lower frequency peak is mainly a function of the transducer resonance as indicated in Fig. 12.

These results give an indication that the model is giving good results in terms of impedance predictions and, by extension through the stainless steel results, the other outputs will also be reliable. Now it is necessary to analyse the predicted separation performance. For this the stored energy frequency product is the preferred variable, and the output from the model for this parameter is given below in Fig. 13.
In comparison with the original best estimate predictions given in Fig. 6, it can be seen that the peak stored energy frequency product number is greater than the original predictions (but of the same order of magnitude), but the peak occurs at a different frequency. This is, in the main, due to the cavity depth being over etched to $0.29 \mathrm{~mm}$ rather than the nominal $0.25 \mathrm{~mm}$. The results, however, indicate that the device will function as a separator.

\section{Controller}

In order for the device to function, it must be driven at the higher frequency impedance null (shown in Fig. 12) at about 


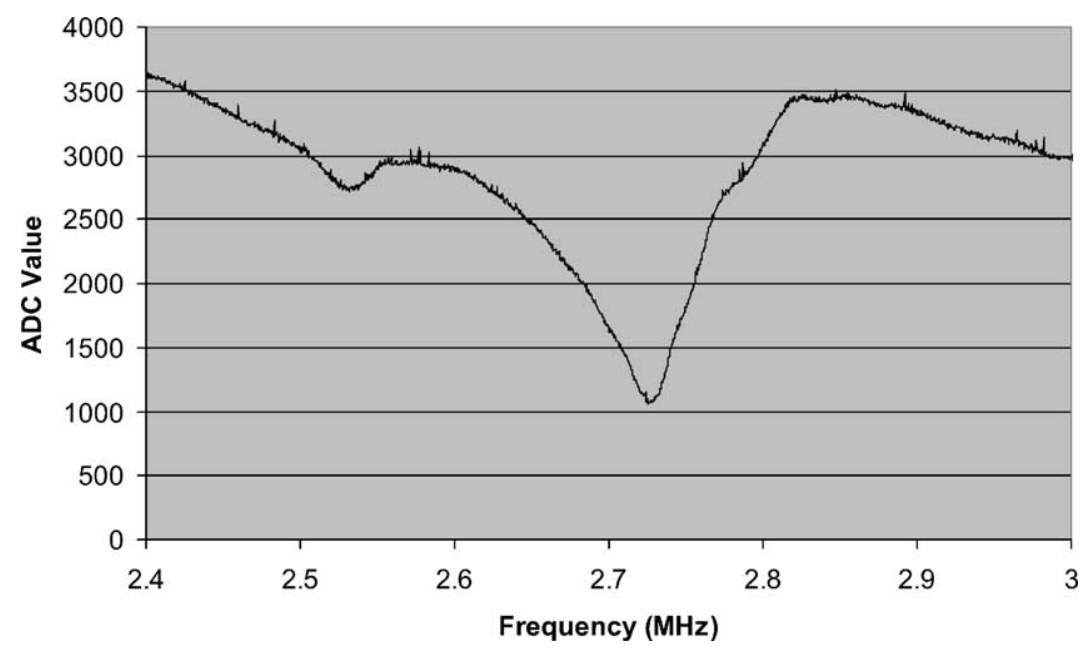

Fig. 15. Example of the voltage trace seen by the controller.

2.75 MHz. This coincides with the peak of the energy storage in the chamber. As already indicated, the frequency of this null may move around slightly, as the fluid temperature, or the concentration of particles may alter. This requires some form of closed loop controller. It is possible to implement a flexible controller using a microcontroller, which closes the loop by sampling the transducer voltage, and using this to make adjustments to a voltage-controlled oscillator. The construction of a flexible controller such as this also allows the transducer voltage information to be extracted, together with the frequency information, hence allowing detailed monitoring and data logging of these parameters. A system diagram of the controller is given in Fig. 14 and gives an indication of the flexibility of the system. In particular a two line LCD display has been incorporated, allowing local indication of parameters such as frequency or voltage measurements, as well as an RS232 serial interface allowing PC control and data logging.

The system is programmed in $\mathrm{C}$, allowing rapid implementation of programs. Fig. 15 shows an example output of the analogue to digital converter raw value against frequency, as measured by the controller and logged to a PC. The cavity resonance can clearly be extracted as it corresponds to a null in the measured voltage across the cell at about $2.73 \mathrm{MHz}$ in this case.

\section{Conclusions}

A micromachined microfluidic ultrasonic separator has been modelled, designed and built using standard micromachining processes. The results from the model compare favourably with the results obtained from a previous separator built in stainless steel. By a process of measuring impedance, and using the results to refine the model a layer at a time, it has been possible to produce a model that gives high confidence of predicting sepa- rator performance. A flexible digital controller has been designed and built and is able to identify the operation point. Work is ongoing to test the separation performance of the device and develop the concept to include a thick-film PZT actuator rather than the bulk PZT currently used.

\section{Acknowledgements}

The authors wish to thank the Engineering and Physical Research Council (EPSRC) for their financial support under grant number GR/R13333/01. We also gratefully acknowledge the Financial support given by Dstl and Microfiltrex Ltd. and the assistance of Dr. Christian Maul of TaiCaan Technologies for the use of their non-contact 3D profiling system.

\section{References}

[1] M. Groschl, Ultrasonic separation of suspended particles. Part I. Fundamentals, Acustica 84 (3) (1998) 432-447.

[2] J.J. Hawkes, W.T. Coakley, Force field particle filter, combining ultrasound standing waves and laminar flow, Sens. Actuators B 75 (2001) 213-222.

[3] N. Harris, M. Koch, S. Beeby, N.M. White, A.G.R. Evans, Thick-film printing of PZT onto silicon for micromechanical applications, in: Proceedings of the Ninth Micromechanics Europe Workshop, 1998, pp. $78-81$.

[4] M. Hill, Y. Shen, J.J. Hawkes, Modelling of layered resonators for ultrasonic separation, Ultrasonics 40 (2002) 385-392.

[5] M. Hill, R.J.K. Wood, Modelling in the design of a flow-through ultrasonic separator, Ultrasonics 38 (2000) 662-665.

[6] M. Hill, N.R. Harris, R. Townsend, S.P. Beeby, Y. Shen, N.M. White, J.J. Hawkes, A microfluidic device for ultrasonic separation, in: Forum Acusticum, Proceedings of the Third EAA European Congress on Acoustics (Sociedad Espanola De Acustica, Sevilla, 2002), PHA-01-003-IP. 


\section{Biographies}

Nick Harris graduated in 1988 from the University of Bath and was awarded a PhD in 1997 from the University of Southampton, on the use of ultrasound for communication purposes. His research interests include thick-film sensors and surface acoustic wave devices, microengineering, and sensor interfacing. He is now a senior research fellow based in the electronics and computer science department at Southampton. $\mathrm{He}$ is a member of the IEE and a chartered engineer.

Martyn Hill graduated in 1985 from the Institute of Sound and Vibration Research at Southampton and moved to the mechanical engineering department where he researched into parameter estimation techniques and then automotive electronics. He is now a senior lecturer in the school of engineering sciences at Southampton with research interests in ultrasonics and particularly in their applications in Microsystems.

Steve Beeby obtained his BEng(Hons) in mechanical engineering in 1992 and was awarded his $\mathrm{PhD}$ in 1998. He is currently a Research Fellow in the Department of Electronics and Computer Science and is researching in the field of microelectromechanical systems (MEMS). He has been responsible for successfully combining thick-film printed piezoelectrics with micromachined silicon devices for MEMS applications and has also developed MEMS resonant and capacitive sensors. His interests include the finite element modelling and design of MEMS devices, silicon processing and MEMS packaging and testing. He currently has over 40 publications in the field.

Yijun Shen was a research student in the school of engineering sciences, and has recently submitted his thesis for the degree of $\mathrm{PhD}$ in the field of acoustic modelling. His main interest has been in modelling both stainless steel and silicon acoustic separators. He has recently been appointed Teaching Company Associate at the University of Cranfield.

Neil M. White is a professor of intelligent sensor systems within the Department of Electronics and Computer Science at the University of Southampton and also director of the Institute of Transducer Technology. He was awarded a PhD in 1988 for a thesis on the application of thick-film piezoresistors for load cells. Professor White was appointed as lecturer in 1990, senior lecturer in 1999, reader in 2000 and currently holds a Personal Chair. He has published extensively in the area of thick-film sensors and intelligent instrumentation and is author or co-author of over one hundred scientific publications. He is a fellow of the Institute of Physics, a chartered engineer and a senior member of the IEEE and has served of several committees in various professional bodies.

Jeremy J. Hawkes gained a degree in physiology and biophysics from The University of East Anglia in 1981 and a $\mathrm{PhD}$ in bioelectronics from UCNW Bangor in 1988. Since then he has been working to develop two cell manipulation methods based on ultrasound standing waves and dielectrophoresis. He is now working in the Departmet for Instrumentation and Analytical Science at UMIST. His interests are in the study of biological systems through their interaction with oscillating force fields and particularly for reagent-free characterization of cells.

W.T. Coakley obtained an MSc degree in experimental physics at University College Cork in 1963, and a PhD in microbiology in University of Wales Cardiff in 1971. He is now professor in the Bioultrasonics Lab in the School of Biosciences at Cardiff University. His interest include ultrasonic deposition of material on biosensors, acoustic microstreaming, membrane poration and cell adhesion. 